\title{
A Case of Suspicious Encephalitis Lethargica Accompanied by Visual Acuity Decrement
}

\section{Joon Hyun Baek and Kee Hyung Park}

Department of Neurology, Gil Medical Center, Gachon University, Incheon, Republic of Korea

${ }^{*}$ Corresponding author: Kee Hyung Park, Department of Neurology, Gil Medical Center, Gachon University, 1198 Guwoldong, Namdong-Gu, Incheon, 405-760 Republic of Korea, Tel: +82-32-460-3346; Fax:+82-32-460-3340; E-mail: khpark@gachon.ac.kr

Rec date: Aug 04, 2014, Acc date: Aug 10, 2014, Pub date: Aug 13, 2014

Copyright: $\odot 2014$ Baek, et al. This is an open-access article distributed under the terms of the Creative Commons Attribution License, which permits unrestricted use distribution, and reproduction in any medium, provided the original author and source are credited.

\begin{abstract}
Encephalitis lethargica (EL) is a CNS disorder presenting with pharyngitis followed by sleep disorder, basal ganglia signs (particularly parkinsonism), and neuropsychiatric sequelae. It was first described in 1916, but only sporadic cases have been described since the 1916-1927. EL is a rare disease and difficult to confirm because no diagnostic criteria have been agreed. Its additional common features include oculogyric crises and ocular features (ophthalmoplegia and ptosis). However, no case of decreasing visual acuity has been previously reported. We report a case of EL with accompanying bilateral blindness. A 31-year-old female patient visited our emergency room complaining of a headache, fever, pharyngitis, and poor oral intake. After the fever and headache subsided, the patient experienced drowsiness and bilateral decreases in visual acuity.
\end{abstract}

Brain magnetic resonance imaging (MRI) showed increased signal intensity on the hypothalamus on fluid attenuated inversion recovery (FLAIR) and T2 weighted images, which was compatible with EL. Although we critically discuss other potential etiologies, we conclude that her blindness constitutes an unusual presentation of EL.

Keywords: Encephalitis lethargica; Encephalitis; Narcolepsy; Polysomnography; Multiple sleep latency test; Von economo; Decrement of visual acuity; VEP

\section{Introduction}

Encephalitis lethargica (EL) is a neurologic complication that may result from influenza or an immune-mediated disease. Other than sleep disturbance, lethargy and extrapyramidal movements are characteristic of EL. Von Economo noted neuropsychiatric disorders (e.g., catatonia, obsessive or compulsive disorder, and mutism) were common in survivors. Other common features included oculogyric crises, ocular features (ophthalmoplegia and ptosis) and central cardiorespiratory features (particularly hiccoughs) [1]. MRI of the brain was normal in $60 \%$ but showed inflammatory changes localized to the hypothalamus in $40 \%$ of patients. 1 However, no of associated loss of vision has been previously reported.

Our patient developed loss of vision progressively in a short time, which could not be explained by ocular examination or a brain lesion. This raised the possibilities of other underlying etiologies, but her clinical symptoms, polysomnography, and MRI findings suggested that the bilateral blindness was an unusual complication of EL.

\section{Summary of Case}

A 31 year-old female presented at our centre with headache and fever. She had suffered from a headache for about 3 weeks, and 2 weeks previously, her symptoms were aggravated by nausea and vomiting. She also complained of right monocular diplopia and visual dimness in both eyes for around a week. Brain MRI has been performed at other hospital but no remarkable finding resulted. In addition, she developed a fever (up to $38^{\circ} \mathrm{C}$ ) 2 days before this presentation and became delirious. Anterograde amnesia was also noticed a day before admission. During the 3 weeks prior to presentation, she had eaten little and had lost $7 \mathrm{~kg}(167 \mathrm{~cm}, 51 \mathrm{~kg}$, body mass index $18.29 \mathrm{~kg} / \mathrm{m}^{2}$ ). There was no relevant medical history and she rarely drank alcohol.

On admission, her orientation was intact but she showed abnormal behaviors, such as, smiling alone, and a slightly lethargic mental status. She still complained of visual dimness in both eyes and diplopia but no abnormal finding, such as, papilledema or retinal hemorrhage was found by fundoscopy. No other abnormal neurologic sign was noted.

Blood analysis indicated hypoosmolar hyponatremia (osmolality: $242 \mathrm{mOsm} / \mathrm{kg}$, sodium: $121 \mathrm{mEq} / \mathrm{L}$ ) with mild elevations of amylase and lipase (amylase: $126 \mathrm{U} / \mathrm{L}$, lipase: $111 \mathrm{U} / \mathrm{L}$ ) Her serum thiamine level was $147.6 \mathrm{nmol} / \mathrm{L}$, which is in a normal range. Spinal tapping revealed elevated WBC and protein (pressure: $10 \mathrm{~cm} \mathrm{H2O,} \mathrm{RBC:} \mathrm{3,}$ WBC: 111, cerebrospinal fluidglucose: $66 \mathrm{mg} / \mathrm{dl}$, Serum glucose: 109 $\mathrm{mg} / \mathrm{dl}$, protein: 47.8 ). Brain MRI showed increased signal intensity on hypothalamus on FLAIR and T2W images without contrast enhancement or diffusion restriction. Electroencephalography (EEG) showed no epileptiform discharge (Figure 1).

Due to the possibility of Wernicke encephalitis, thiamine $50 \mathrm{mg}$ twice a day was infused for 10 days, and IV acyclovir was administered for more than 2 weeks due to the possibility or herpes encephalitis. However, no interval changes in her clinical symptoms were noted.

Other components of the differential diagnosis were dengue infection, multiple sclerosis (MS), tuberculosis (Tb), meningitis, and Japanese encephalitis. We ruled out a dengue infection because it usually occurs in tropical or subtropical regions and because her white blood cell counts were normal. In addition, a tourniquet test showed no petechial hemorrhages. Multiple sclerosis was also ruled out 
because of the absence of any brain lesion and of any space disseminated MS lesion. Tb meningitis was viewed unlikely because acid bacillus (AFB) stain, culture, and Tb PCR of CSF (cerebrospinal fluid) were negative and CSF adenosine deaminase was not increased.
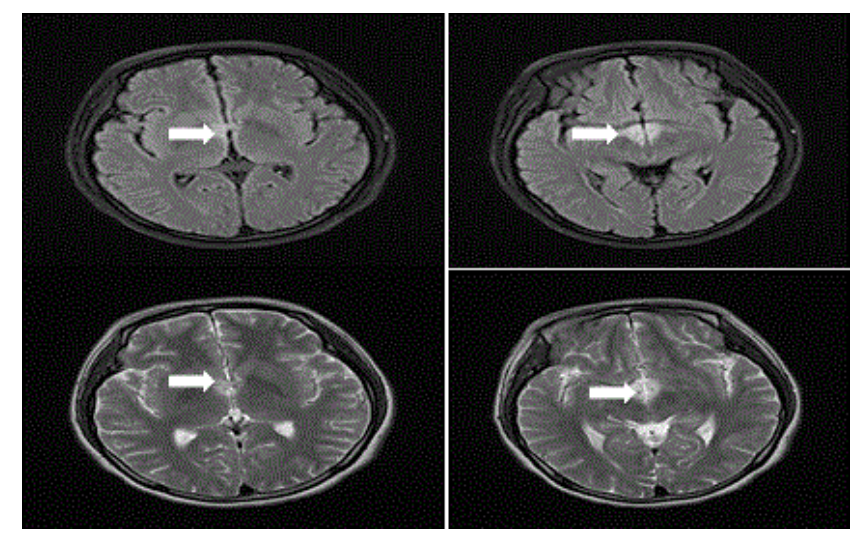

Figure 1: MRI images without gadolium contrast injection. High signal intensities were observed in the mammillary body and fornix on Fluid attenuated inversion recovery (FLAIR) images and on T2 weighted images without contrast enhancement or diffusion restriction (White arrow).

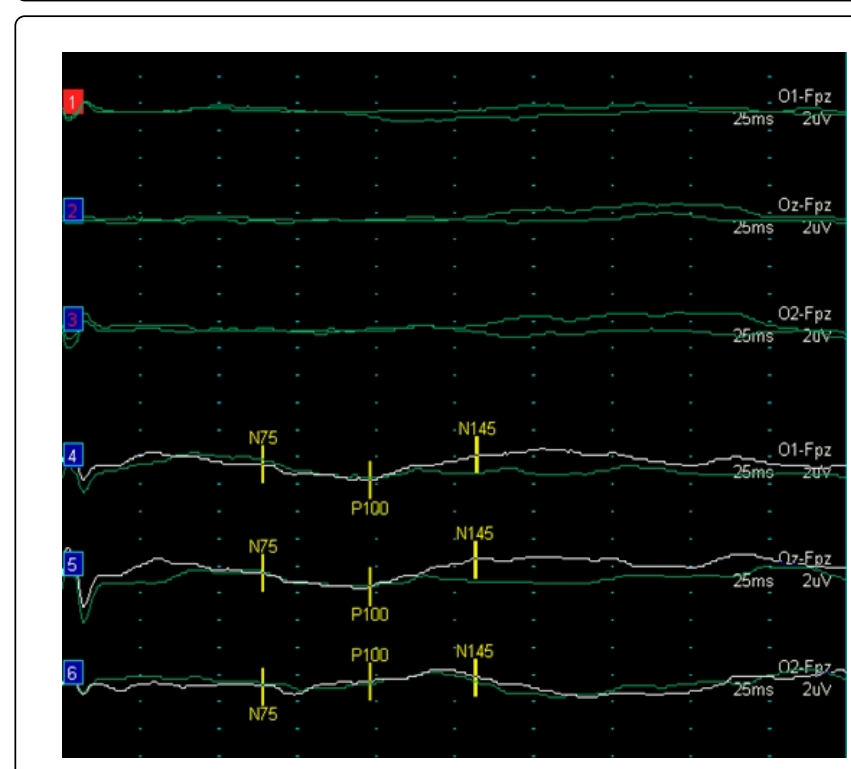

Figure 2: Abnormal findings of Visual evoked potentials (VEPs) No P100 wave was detected at the left eye by Visual evoked potentials (VEPs) which indicates left optic nerve injury.

On day 5, her fever subsided but she remained drowsy and her visual acuity worsened. Direct light reflex was absent but indirect light reflex was present in the left eye, whereas both were present in the right eye. No P100 wave was detected on her left eye on visually evoked potentials (VEP), indicating the possibility of a lesion on her left optic nerve or eye (Figure 2). On day 10, follow up brain MRI revealed no interval change, and spinal tapping still showed pleocytosis and a high level of protein (pressure: $12 \mathrm{~cm} \mathrm{H}_{2} \mathrm{O}, W B C$ : 102 , protein: 62.4 , glucose $57 / 104$ ).
A week later, she only stayed awake for about 5 to 10 minutes at mealtimes. Sleep polysomnography was checked due to her drowsy mental state and it showed abnormal sleep architecture. Sleep latency was 1 minutes and sleep efficiency was $84.30 \%$. Her arousal index was 23.4 /hour, wakefulness after sleep onset (WASO) was $15.59 \%$, apnea/ hypopnea index was 0 per hour of sleep. On the same day multiple sleep latency testing (MSLT) was performed. Sleep latency shorted to 12 seconds. In addition, Sleep Onset Rapid EYE Movement (SOREM) was observed during each MSLT test (Figure 3). We started 'Modafinil' at $200 \mathrm{mg}$ per day and her symptoms improved slightly (Tables 1 and 2).

Although mild improvements in mental state and other general symptoms, such as, fever and headache, were observed, she remained drowsy and her visual acuity did not improve. The patient discharged and asked to maintain her medication.

\section{Discussion}

In recent years, encephalitis lethargica, as described by Von Economo, has become a rare disease. Previously Von Economo described three main types of acute illness during the pandemic that range from 1914 to 1930, which was of the somnolentophthalmoplegic form, the akinetic-mute form, or the hyperkinetic form [2].

Although several case reports of sporadic encephalitis lethargica have been described in the last 30 years, details of the acute phase have often been poorly documented despite the fact that the diagnosis is reliant on its clinical features. Rail et al. [3] provided a detailed account of the acute illness, which concurred well with the original description of Von Economo, and outlined several clinical features that help to distinguish encephalitis lethargica from other encephalitides associated with extrapyramidal syndrome as, an encephalitic illness with parkinsonian features developing acutely or after a oculogyric crises or alteration in sleep cycle or ocular or pupillary changes, or involuntary movements (e.g., dystonia, myoclonus), mental changes, or corticospinal tract signs.

Howard and Lees reinforced these criteria and defined encephalitis lethargica as an acute or subacute encephalitic illness including at least three of the following criteria, after excluding all other known causes of encephalitis: (i) signs of basal ganglia involvement, (ii) oculogyric crises, (iii) ophthalmoplegia, (iv) obsessive-compulsive behavior, (v) akinetic mutism, (vi) central respiratory irregularity, and (vii) somnolence and/or sleep inversion. In addition, he added further investigative criteria, such as abnormalities of cerebrospinal fluid (CSF; lymphocytosis, increased protein levels, oligoclonal bands), EEG changes (diffuse slow wave abnormalities), and visual evoked potential abnormalities [4].

Our case patient did not meet the diagnostic criteria but had sufficient extra symptoms and abnormal investigative findings to suspect EL. Her first symptoms were a headache and fever, and disease progression, daytime sleepiness developed. She also showed ocular symptom, such as, ophthalmoplegia and decrement of visual acuity with abnormal VEP findings. Brain MRI showed increased signal intensity on the hypothalamus of FLAIR and T2W images, which is related with consciousness. CSF analysis showed pleocytosis and protein elevation. Thus, although her clinical symptoms met only two of the six essential features of EL, her distinctive somnolent and ophthalmoplegic symptom and abnormal brain MRI, CSF, and VEP findings suggested an unusual form of EL. 


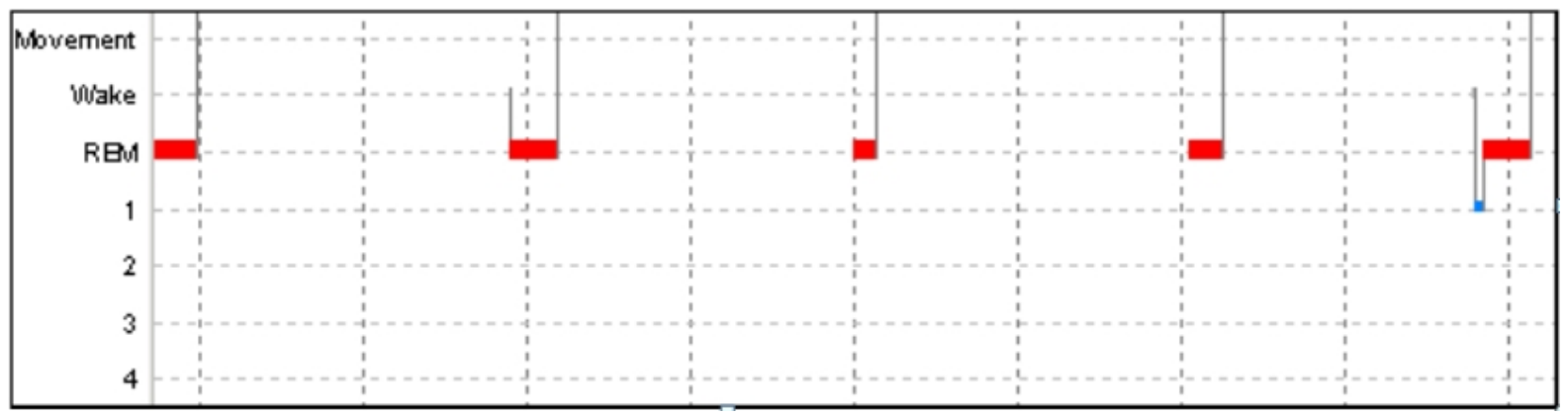

Figure 3: Results for the five naps in the MSLT test. Total five sleep-onset REM periods (SOREMPs) are observed. Time in X-axis shows the clock time during the different hours of the day. Y-axis shows each stage of the sleep. Red box indicates REM sleep.

\begin{tabular}{|l|l|}
\hline & Time \\
\hline Total sleep time & $341 \mathrm{~min}$ \\
\hline Stage 1 sleep & $76.5 \%$ \\
\hline Stage 2 sleep & $4.8 \%$ \\
\hline Slow wave sleep & $0 \%$ \\
\hline REM sleep & $18.6 \%$ \\
\hline Total movement time & 0 min \\
\hline WASO & 63 min \\
\hline Latency to onset & $1 \mathrm{~min}$ \\
\hline Latency to REM sleep & 0 min \\
\hline Sleep efficiency & $84.30 \%$ \\
\hline PLM index & 11.8 per hour \\
\hline Abrevaton: REM: Rapi Eye Movent WASO Wak Aft Seep Onst;
\end{tabular}

Abbreviation: REM: Rapid Eye Movement; WASO: Wake After Sleep Onset; PLM: Periodic Limb Movement Sleep latency is from light out to the first epoch of sleep

Table 1: All-night polysomnographic data obtained at the night before MSLT test. The patient shows frequent arousal, periodic limb movements in sleep, and abnormal sleep architecture in the polysomnography.

Furthermore, as compared with previously reported EL cases, our patient had previously unreported neurologic symptoms. In particular, she had distinctive ocular symptoms, that is, decrement of visual acuity, a pupillary light abnormality, and diplopia. Although several reports have described pupillary light abnormality and diplopia in EL patients, 1 damaged visual acuity has not been reported to date. The absence of left P100 waves in the VEP study indicated actual damage to the left optic nerve, the reason for which was unclear. We suppose the hypothalamic brain lesion, which was near the course of the optic nerve or infection of unknown origin affected the left optic nerve and damaged left visual acuity.

The etiology of the EL has not been resolved. For some time it was thought to be associated with influenza virus, and although an organism or virus has never been isolated, other encephalitic illnesses caused by identified organisms or viruses, such as, coxsackie B2, arborvirus, Japanese B, western equine, measles, varicella, polio, and mycoplasma, are known to be associated with extrapyramidal syndromes [5-8]. On the other hand, a recent examination of archived EL brain material failed to demonstrate influenza RNA, and thus, supported the notion that EL is not an invasive influenza encephalitis [1]. By contrast, the findings of intrathecal oligoclonal bands (OCB), the causative role of Anti-NMDA-receptor (NMDAR), and the known beneficial effects of steroid treatments favor the hypothesis that EL is immune-mediated. 1,8 Our patient had a preceding viral-like infection before clinical symptoms development and had pleocytosis in CSF fluid. In addition, her symptoms did not improved on steroid. These infectious symptoms, CSF abnormality, and the ineffectiveness of steroid support the classical hypothesis of the etiology of EL. Further studies on NMDAR are needed to facilitate the diagnosis and efficient treatment of EL.

Our patient had daytime sleepiness, which is a core symptom of EL, and seems similar to secondary narcolepsy. The diagnosis of narcolepsy is essentially clinical and its estimated incidence varies across countries and ethnic groups from 168 to 799 per 100,000 in most studies, although Japanese studies have indicated a prevalence as high as 1600 per $100,000[9,10]$. The incidence of secondary narcolepsy in EL has not been reported but is likely to be scarce. We found some reports of secondary narcolepsy related to the infection but unfortunately sleep has rarely been analyzed in infectious patients, due to the emergency aspects of diseases like meningitis or severe viral infection. In previous studies sleep modifications associated with bacterial or viral diseases have been linked with immune reactions. The nature of the uncoupling between the acute phase of the immune reaction in sleeping sickness and sleep-wake patterns has not been determined [11].

Nocturnal polysomnography followed by a Multiple Sleep Latency Test (MSLT) is a useful tool for diagnosing narcolepsy. The diagnostic criterion of narcolepsy is a mean sleep latency of less than eight minutes with at least two sleep onset REM periods (SOREMPs). Our polysomnography study showed frequent arousal, periodic limb movements in sleep, and an abnormal sleep architecture that increased in stage I and decreased in stage II, SWS, and REM sleep. Also in the MSLT study, sleep latency was shortened to 12 seconds and SOREMPs were observed all five trials. Accordingly, her MSLT results were compatible with narcolepsy. 
Page 4 of 4

\begin{tabular}{|c|c|c|c|c|c|}
\hline \multicolumn{2}{|c|}{ Session } & \multicolumn{4}{|c|}{ Time(minutes) } \\
\hline & TST & NREM & REM & Sleep latency & REM sleep \\
\hline 1. & 15.0 & 0.0 & 15.0 & 0.4 & 0.4 \\
\hline 2. & 17.5 & 0.0 & 17.5 & 0.5 & 0.5 \\
\hline 3. & 8.0 & 0.0 & 8.0 & 0.3 & 0.3 \\
\hline 4. & 12.5 & 0.0 & 12.5 & 0.0 & 0.1 \\
\hline 5. & 21.0 & 0.0 & 18.0 & 0.5 & 3.5 \\
\hline
\end{tabular}

Table 2: MSLT results for the patient. Sleep latency was reduced to 12 seconds and SOREMPs were observed in all five MSLT trials.

As far as we know this is the first polysomnography study of an EL patient, and consider it provides a basis for the identification of the sleep patterns of EL and secondary narcolepsy related to infection. We assume that the sleep pattern of EL shares the features of that of narcolepsy, as exhibited by our patient. Because somnolence is distinctive feature of EL and polysomnography is easily accessible and provides a non-invasive way of diagnosing narcolepsy, we hope our findings aid the understanding of the pathophysiology of EL and aid future diagnoses (Tables 1 and 2).

The brain lesion depicted by MRI in our patient may explain her hypersomnia. In 1916 Von Economo first described hypersomnia patients with a brain lesion in the posterolateral hypothalamic region of the brain, which regulates sleep and wakefulness [12]. The ascending arousal system largely originates from a series of welldefined cell groups with identified neurotransmitters. This pathway has two major branches. The first branch is an ascending pathway to the thalamus that activates the thalamic relay neurons crucial for transmission of information to the cerebral cortex, whereas the second branch bypasses the thalamus, and instead activates neurons in the lateral hypothalamic area, basal forebrain (BF), and throughout the cerebral cortex. Lesions along this second pathway, particularly in the lateral hypothalamus (LHA) or rostral midbrain, produce the most profound and long-lasting forms of sleepiness or even coma [12].

Recently, several symptomatic narcoleptic cases were described with unique bilateral symmetric hypothalamic lesions associated with significant hypocretin ligand deficiency [10]. Accordingly, given reported cases and the anatomy and functions of these systems, it is not surprising that our patient, who had a lesion on the hypothalamus, showed profound and long-lasting arousal impairment [13].

We report a rare case of EL with accompanying bilateral blindness. No definitive diagnostic criteria have been agreed for EL, but some effort has been expended to determine its pathophysiology. EL is an extremely rare disease and few cases have been reported. Although our patient was without extrapyramidal symptoms and a definite neuropsychiatric symptom, its distinctive somnolent-ophthalmoplegic form, which is a common presentation, and abnormal brain MRI findings, which are compatible with a previous report of Von Economo, and abnormal CSF study supports a diagnose of an unusual form of EL. Furthermore, we report two new findings not previously associated with EL. First, our patient exhibited decrement of visual acuity, which we believe may be new symptom of EL. Second, we performed polysomnography to obtain some information about the sleeping pattern of EL, and as far as we know this is the first polysomnography report in EL and hope the information provided aids the characterization of its sleep pattern.

\section{References}

1. Dale RC, Church AJ, Surtees RA, Lees AJ, Adcock JE, et al. (2004) Encephalitis lethargica syndrome: 20 new cases and evidence of basal ganglia autoimmunity. Brain 127: 21-33.

2. Newman KD (1931) Translation of Von Economo. Encephalitis lethargica: its sequelae and treatment. London.

3. Rail D, Scholtz C, Swash M (1981) Post-encephalitic Parkinsonism: current experience. J Neurol Neurosurg Psychiatry 44: 670-676.

4. Howard RS, Lees AJ (1987) Encephalitis lethargica. A report of four recent cases. Brain $110: 19-33$.

5. Poser CM, Huntley CJ, Poland JD (1969) Para-encephalitic parkinsonism. Report of an acute case due to coxsackie virus type B 2 and re-examination of the etiologic concepts of postencephalitic parkinsonism. Acta Neurol Scand 45: 199-215.

6. Duvoisin RC, Yahr MD (1965) ENCEPHALITIS AND PARKINSONISM. Arch Neurol 12: 227-239.

7. Al-Mateen M, Gibbs M, Dietrich R, Mitchell WG, Menkes JH (1988) Encephalitis lethargica-like illness in a girl with mycoplasma infection. Neurology 38: 1155-1158.

8. Tan A, Shuey N, Bladin C (2010) A modern perspective on the differential diagnosis between encephalitis lethargica or anti-NMDAreceptor encephalitis. J Clin Neurosci 17: 1204-1206.

9. Longstreth WT Jr, Koepsell TD, Ton TG, Hendrickson AF, van Belle G (2007) The epidemiology of narcolepsy. Sleep 30: 13-26.

10. Tashiro T, K anbayashi T, Iijima S, Hishakawa $Y$ (1992) An epidemiological study on prevalence of narcolepsy in Japanese. J Sleep Res (Suppl 1): 228.

11. Dauvilliers Y, Buguet A (2005) Hypersomnia. Dialogues Clin Neurosci 7: 347-356.

12. Saper CB, Scammell TE, Lu J (2005) Hypothalamic regulation of sleep and circadian rhythms. Nature

13. Kanbayashi T, Sagawa Y, Takemura F, Ito SU, Tsutsui K, et al. (2011) The pathophysiologic basis of secondary narcolepsy and hypersomnia. Current Neurology and Neuroscience Reports 11: 235-241. 